\title{
Multimode Dynamics and Emergence of a Characteristic Length Scale in a One-Dimensional Quantum System
}

\author{
M. Kuhnert, ${ }^{1}$ R. Geiger, ${ }^{1}$ T. Langen, ${ }^{1}$ M. Gring, ${ }^{1}$ B. Rauer,${ }^{1}$ T. Kitagawa, ${ }^{2}$ \\ E. Demler, ${ }^{2}$ D. Adu Smith, ${ }^{1}$ and J. Schmiedmayer ${ }^{1, *}$ \\ ${ }^{1}$ Vienna Center for Quantum Science and Technology, Atominstitut, TU Wien, Stadionallee 2, 1020 Vienna, Austria \\ ${ }^{2}$ Department of Physics, Harvard-Massachusetts Institute of Technology Center for Ultracold Atoms (CUA), \\ Harvard University, Cambridge, Massachusetts 02138, USA \\ (Received 22 November 2012; published 28 February 2013)
}

\begin{abstract}
We study the nonequilibrium dynamics of a coherently split one-dimensional Bose gas by measuring the full probability distribution functions of matter-wave interference. Observing the system on different length scales allows us to probe the dynamics of excitations on different energy scales, revealing two distinct length-scale-dependent regimes of relaxation. We measure the crossover length scale separating these two regimes and identify it with the prethermalized phase-correlation length of the system. Our approach enables a direct observation of the multimode dynamics characterizing one-dimensional quantum systems.
\end{abstract}

The nonequilibrium dynamics of many-body quantum systems and their pathway towards equilibrium is of fundamental importance in vastly different fields of physics. Open questions appear, for example, in high-energy physics for understanding quark-gluon plasma [1-3], in cosmology for describing preheating of the early Universe [4], or in the comprehension of relaxation processes in condensed-matter systems [5,6].

Because of their isolation from the environment and their tunability, ultracold atom systems have triggered many studies of nonequilibrium dynamics in closed interacting quantum systems, with particular interest drawn to quantum quenches $[7,8]$. Important questions are related to systems where the dynamics is constrained by several constants of motion [9] and to the possible description of nonequilibrium states by generalized statistical mechanics ensembles $[10,11]$.

Recently, we reported the experimental observation of prethermalization in a coherently split one-dimensional (1D) ultracold Bose gas [12], made possible by a characterization of the dynamical states through measurements of full distribution functions [13,14]. Prethermalization [15] was understood as the rapid relaxation to a steady state exhibiting thermal-like properties but differing from the true thermal equilibrium that is eventually expected to occur on longer time scales [16-20].

In this Letter, we study the relaxation process [21] leading to the prethermalized state by measuring the full (probability) distribution functions (FDFs) of phase and

Published by the American Physical Society under the terms of the Creative Commons Attribution 3.0 License. Further distribution of this work must maintain attribution to the author(s) and the published article's title, journal citation, and DOI. contrast of matter-wave interference. We probe the $1 \mathrm{D}$ system on different length scales to investigate its multimode dynamics, which reveals two distinct regimes separated by a characteristic crossover length scale. We measure this characteristic length scale and identify it with the effective thermal phase-correlation length of the prethermalized system.

We prepare a quasi-1D Bose gas of several thousand ${ }^{87} \mathrm{Rb}$ atoms in an elongated (along the $z$ direction) magnetic microtrap on an atom chip [22] at a (tunable) temperature between 20 and $120 \mathrm{nK}$. The gas is coherently split along the radial $(x)$ direction using a symmetric radio-frequency dressed-state double-well potential [23], creating two uncoupled 1D gases separated by a distance of $3.1 \mu \mathrm{m}$ [Fig. 1(a)]. The longitudinal and radial trap frequencies in the double well are $7 \mathrm{~Hz}$ and $1.4 \mathrm{kHz}$, respectively, and the size of the system in the longitudinal direction is typically $100 \mu \mathrm{m}$ (see Supplemental Material [24]). The system is then held in the double-well trap for a variable evolution time $t_{e}$ before being released and allowed to fall under gravity during a time of flight of $16 \mathrm{~ms}$. The resulting matter-wave interference pattern [Fig. 1(b)] is recorded using absorption imaging [25] and contains information about the local relative phase $\phi(z)=\theta_{1}(z)-\theta_{2}(z)$ between the two halves of the system, with $\theta_{1,2}(z)$ being the fluctuating phase profile of each individual gas.

In 1D systems, spatial fluctuations arising from excitations at different wavelengths strongly affect the physics [26], and probing the system on variable lengths $L$ represents a filter for the effects of these excitations [27]. In our experiment, we integrate the interference pattern longitudinally over a length $L$ and extract a line profile from which a contrast $C(L)$ and a phase $\Phi(L)$ are obtained [Fig. 1(c)]. Repeated realizations allow us to measure the FDF of phase and contrast for different evolution times 


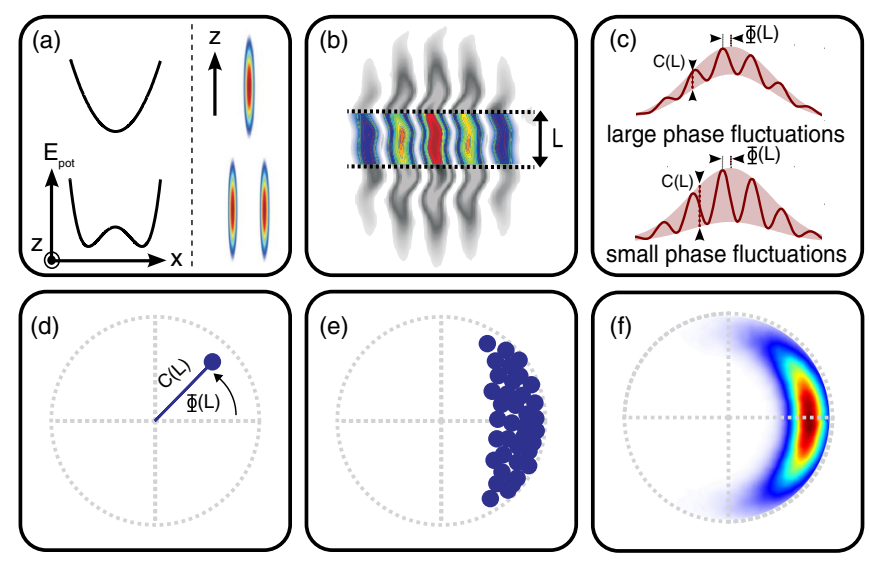

FIG. 1 (color online). Matter-wave interferometry and correlation measurements. (a) The experiment is initiated by coherently splitting a single quasi-1D Bose gas into two uncoupled gases using a horizontal double-well potential. The system is then held to evolve for a variable evolution time $t_{e}$ before being released from the trapping potential. The contrast of the resultant interference pattern is a direct measure of the relative phase fluctuations (b). Integrating the interference pattern over a length $L$ and fitting the resulting line profile (c) gives the phase $\Phi(L)$ and the contrast $C(L)$, which are plotted as circular statistics (d). (e) The whole process is repeated many times (typically 150) to map the FDF for a particular length scale $L$ and evolution time $t_{e}$. The point plot in (e) is then converted into a density plot (f).

$t_{e}$ and integration lengths $L$. Our measured FDFs are presented in Fig. 2.

For the shortest evolution time (1.5 ms in Fig. 2), we observe high contrasts and small phase spreads for all lengths $L$, which demonstrates the coherence of our splitting process. After splitting, the phase fluctuations corresponding to the different excitations grow in magnitude, resulting in a scrambling of the relative phase field $\phi(z)$. These spatial fluctuations of $\phi(z)$ manifest themselves in a randomization of the phase in the interference patterns and in a decrease of the contrast, which depends on the probed length scale $L$. For short $L$ values, the sparsely populated high-momentum modes satisfying $k>2 \pi / L$ do not lead to contrast reduction, resulting in a ringlike shape of the FDFs; this is the phase-diffusion regime (A in Fig. 2). For long $L$ values, many modes satisfying $k>2 \pi / L$ are populated and their dynamics leads to a scrambling of $\phi(z)$ within the probed integration length, resulting in a significant reduction of the probability of observing a high contrast and a disklike shape of the FDFs; this is the contrast-decay regime (B).

At increasingly longer evolution times $\left(t_{e}>10 \mathrm{~ms}\right)$, the FDFs do not change significantly, revealing the emergence of a steady state characterized by a crossover length scale between the phase-diffusion (A) and contrast-decay (B) regimes. In our previous work [12], we identified this steady state as a prethermalized state.

We now quantitatively analyze our observations in the framework of an integrable Luttinger liquid (LL)

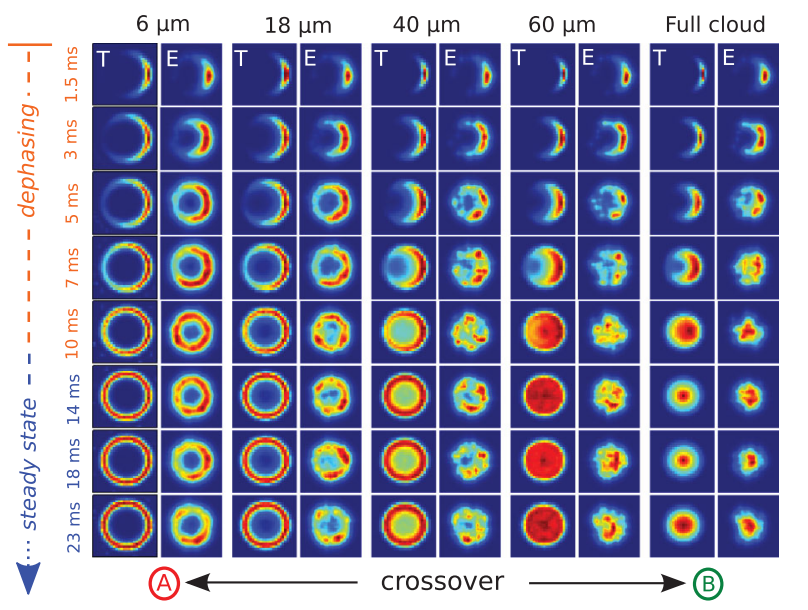

FIG. 2 (color). Multimode dynamics revealed by FDFs of matter-wave interference. The probability density of contrast $C(L)$ and phase $\Phi(L)$ of interference patterns is measured for each integration length $L$ (horizontal axis) and evolution time $t_{e}$ (vertical axis). Red and blue denote high and low probability, respectively, and the color map is rescaled for each plot. For each value of $L$, the right (left) columns correspond to the experimental data (theoretical calculations) and full cloud to $L=$ $100 \mu \mathrm{m}$ [33]. At $t_{e}=1.5 \mathrm{~ms}$, the high contrasts and small phase spreads demonstrate the coherence of the splitting process. As time evolves, a steady state emerges and two distinct lengthscale-dependent regimes appear: the phase-diffusion regime (A) and the contrast-decay regime (B). For short (long) $L$ values, the phase is random and the probability of observing a high contrast is high (low), resulting in a ring (disk) shape in the density plot. The theoretical calculations take into account the technical noise on the relative atom number between the two wells (standard deviation measured to be $5 \%$ of the total atom number) and the error associated with the fitting of the interference patterns.

theory. To describe the dynamics, we consider the LL Hamiltonian

$$
\hat{H}=\frac{\hbar c}{2} \int_{-L / 2}^{L / 2} d z\left[\frac{K}{\pi}(\nabla \hat{\phi})^{2}+\frac{\pi}{K} \hat{n}^{2}(z)\right]
$$

determining the time evolution of the operators $\hat{\phi}(z)$ and $\hat{n}(z)$ representing the relative phase and the relative density of the system, respectively [21,27]. Here, $c=\sqrt{\rho g / m}$ is the sound velocity, $K=\pi \xi \rho$ is the LL parameter characterizing the strength of the interactions, and $\xi=\hbar / m c$ is the healing length; $\rho$ is the $1 \mathrm{D}$ density in each half of the system, $g=2 \hbar \omega_{\perp} a_{s}$ is the $1 \mathrm{D}$ coupling constant, $m$ is the mass of the ${ }^{87} \mathrm{Rb}$ atom, $a_{s}$ is the scattering length, and $\omega_{\perp}$ is the radial trapping frequency (see Supplemental Material [24]). The time evolution of $\hat{n}(z)$ and $\hat{\phi}(z)$ can be described in Fourier space by a set of uncoupled harmonic oscillators of collective modes with momentum $k$ and energies $\hbar \omega_{k} \approx$ $\hbar c|k|$, where the LL Hamiltonian is diagonal [14].

The rapid splitting process prepares a coherent superposition of the atoms in the two wells, resulting in high relative density fluctuations $\left\langle\hat{n}_{k}^{2}\right\rangle_{t=0}=\rho / 2$ and small 
relative phase fluctuations $\left\langle\hat{\phi}_{k}^{2}\right\rangle_{t=0}=1 / 2 \rho$ [14,24]. In the experiment, we observe enhanced global phase fluctuations for $t_{e}=1.5 \mathrm{~ms}$ with respect to quantum noise, which are attributed to technical noise on the relative atom number between the two wells. For the dynamics of the contrast, the contribution of this technical noise is, however, negligible compared to that of the initial density fluctuations, for the probed integration lengths $(L \gg \xi)$. Focusing on the contrast thus allows us to directly observe the effect of quantum noise associated with the splitting process through the evolution of the many-body system.

The results of the LL calculations, taking into account our technical noise, are presented in the left columns of Fig. 2. For all length scales and evolution times, we find very good agreement between the experimental and theoretical FDFs. Our approach gives a direct and intuitive visualization of the multimode nature of the dynamics in 1D Bose gases [28].

To study the relaxation of the contrast in more detail, in Fig. 3 we compare the measured mean values of the integrated squared contrast $\left\langle\hat{C}^{2}\left(L, t_{e}\right)\right\rangle$ to the prediction of the LL theory, taking into account the imperfections of our imaging system (see Supplemental Material [24]). We observe that the characteristic time for the decay of the contrast is longer for long $L$ than for short $L$, which is well captured by our model. For a more quantitative comparison, we fit the experimental and theory data by an exponential decay and extract the $1 /$ e time constant. For the experiment, we find characteristic decay times $\tau_{\exp }(L=6 \mu \mathrm{m})=0.53 \pm$ $0.33 \mathrm{~ms}$ and $\tau_{\exp }(L=100 \mu \mathrm{m})=2.89 \pm 0.36 \mathrm{~ms}$ (standard error on fitted parameter). Fitting the theoretical

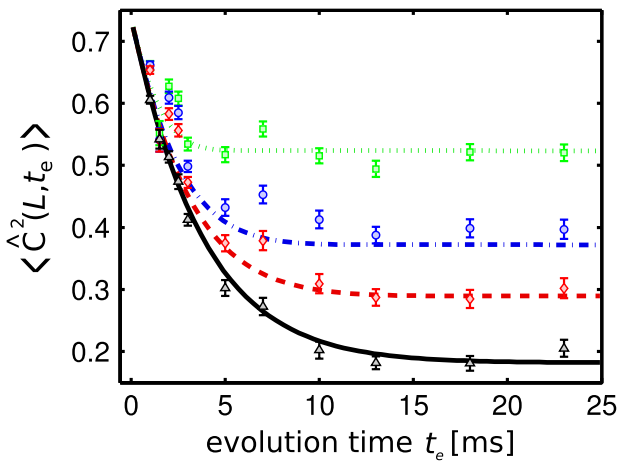

FIG. 3 (color online). Contrast dynamics. Measured values of the mean squared contrast for various integration lengths, corrected for the contrast reduction factor due to the imperfections of our imaging system (see Supplemental Material [24]). From top to bottom: $L=18,40,60,100 \mu \mathrm{m}$. The lines show the results of the Luttinger liquid calculations for these integration lengths. The theory data for all $L$ values have been rescaled by the same factor of $r=0.74$ [34]. Apart from this factor, no fitting parameter is used. We observe a relaxation process in which a steady state is established on a time scale depending on $L$ and corresponding to the dephasing of the different excitations probed within that $L$. calculations for our measured LL parameter $K=58 \pm 4$, we find $\tau_{\text {theo }}(L=6 \mu \mathrm{m})=0.40 \pm 0.11 \mathrm{~ms}$ and $\tau_{\text {theo }}(L=$ $100 \mu \mathrm{m})=3.15 \pm 0.21 \mathrm{~ms}$, in good agreement with the observations. The dependence of the relaxation time scale on $L$ is an additional clear signature of the multimode nature of the dynamics [14].

Figure 3 also reveals the onset of the prethermalized state for $t_{e}>3 \tau \approx 10 \mathrm{~ms}$ which is characterized by high (low) contrasts at short (long) integration lengths, in line with the probability density plots A (B) of Fig. 2. In the rest of this Letter, we investigate the crossover between the high contrasts and low contrasts regimes observed in Figs. 2 and 3.

Within the LL theory, the crossover length scale separating the phase-diffusion and contrast-decay regimes can be calculated analytically [14]: $l_{0}=2 \hbar^{2} / m g=\hbar / m a_{s} \omega_{\perp}$. For the data in Figs. 2 and 3, we measure $\omega_{\perp} / 2 \pi=$ $1.40 \pm 0.08 \mathrm{kHz}$ and calculate $l_{0}=15.8 \pm 0.9 \mu \mathrm{m}$. To understand the nature of this crossover length scale, we consider the decay of the mean squared contrast $\left\langle\hat{C}^{2}(L)\right\rangle$ as a function of $L$ for $t_{e}>10 \mathrm{~ms}$ (inset of Fig. 4). To fit this decay, we use our knowledge of the correlation functions in the prethermalized state, which have been shown to be

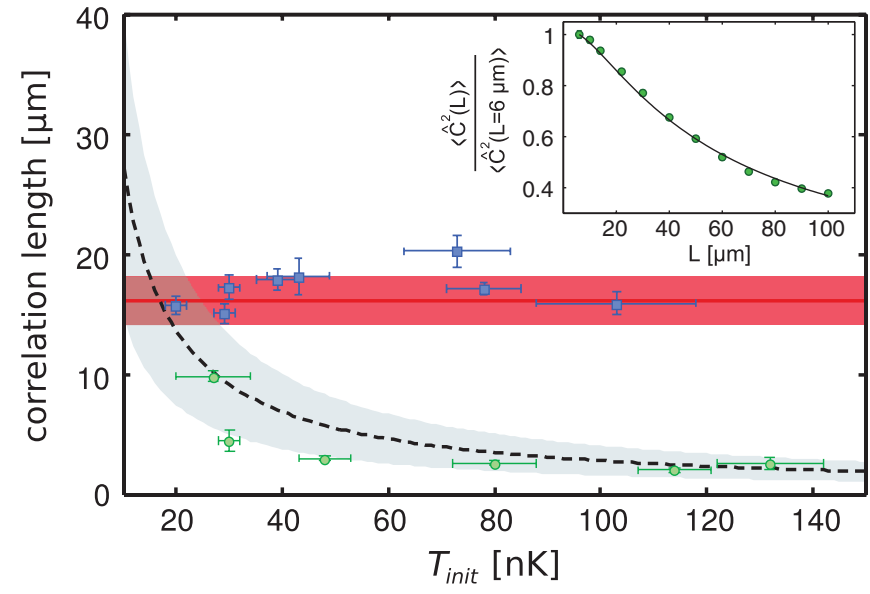

FIG. 4 (color). Comparison of the relative-phase correlation length of the prethermalized system $\left(\lambda_{\text {eff }}\right)$ and of a system at thermal equilibrium $\left(\lambda_{\phi}\right)$. Blue squares: values of $\lambda_{\text {eff }}$ obtained by varying the temperature $T_{\text {init }}$ before splitting the quasicondensate (see main text). Error bars denote 1 standard deviation and are obtained by a bootstrapping method (see Supplemental Material [24]). The red shaded area corresponds to the $95 \%$ confidence interval around the theoretical calculation (line) accounting for the uncertainty on the experimental parameters. Green circles: correlation length $\lambda_{\phi}$ of a system of two quasicondensates in thermal equilibrium, normalized to the mean of the different densities. Gray shaded area: 95\% confidence interval around the theory (dashed line). Inset: estimation of the correlation length. The filled circles are the values of $\left\langle\hat{C}^{2}(L)\right\rangle$ in the prethermalized state (data of Fig. 3 for $t_{e}>10 \mathrm{~ms}$ ), normalized to the value at $L=6 \mu \mathrm{m}$. The solid line is a fit to an effective thermal equilibrium theory with the correlation length being the only free parameter. 
thermal-like [12,14]. Using the direct link between the mean squared contrast and the relative-phase correlation function [24,29], a fit of $\left\langle\hat{C}^{2}(L)\right\rangle$ allows us to estimate the effective thermal phase-correlation length $\lambda_{\text {eff }}$ of the system. For the data in Fig. 4 (inset) we find $\lambda_{\text {eff }}=16.6 \pm$ $0.9 \mu \mathrm{m}$, close to the value of $l_{0}$ calculated above using the independently determined experimental parameters. Note that our method of estimating the correlation length does not require precise measurements of the system parameters to fit the FDFs but only needs the knowledge that the correlation functions are effectively thermal.

The equivalence of the crossover length scale $l_{0}$ and the effective thermal phase-correlation length $\lambda_{\text {eff }}$ can be understood in the following way. In the case of two uncoupled quasicondensates in thermal equilibrium with temperature $T$, the thermal phase-correlation length is given by $\lambda_{\phi}=\hbar^{2} \rho / m k_{B} T$ [30,31]. Due to our rapid splitting process, the energy initially stored in the system is equally distributed between the different $k$ modes of the system $[12,14]$, resulting in thermal-like correlations characterized by an effective temperature $k_{B} T_{\text {eff }}=\left.\langle\hat{H}\rangle\right|_{t=0}=\rho g / 2$. The effective thermal phase-correlation length can thus be identified with $\lambda_{\text {eff }}=\hbar^{2} \rho / m k_{B} T_{\text {eff }}=2 \hbar^{2} / m g$ and is equivalent to the crossover length scale $l_{0}$.

Whereas the prethermalized system reveals thermal-like correlations, its correlation length $\lambda_{\text {eff }}$ depends only on the 1D coupling constant $g$, in contrast to a system of two quasicondensates at thermal equilibrium where $\lambda_{\phi}$ is a function of density and temperature. To reveal this difference experimentally, we varied the initial temperature $T_{\text {init }}$ of the quasicondensate before splitting and measured the correlation length by fitting the decay of $\left\langle\hat{C}^{2}(L)\right\rangle$ (see inset of Fig. 4). The temperature before splitting, $T_{\text {init }}$, was obtained through measurements of the second-order correlation function of longitudinal density fluctuations after time of flight [24,32]. The results (blue squares in Fig. 4) show the independence of $\lambda_{\text {eff }}$ from $T_{\text {init }}$. When varying $T_{\text {init }}$, we further observed that the evolution of the FDFs remained close to that presented in Fig. 2. In particular, the steady states reached in the evolution exhibited the same crossover between the phase-diffusion and contrast-decay regimes. These observations confirm our interpretation of the crossover length scale $l_{0}$ as the prethermalized phasecorrelation length $\lambda_{\text {eff }}$ of the system. Performing experiments with two quasicondensates in thermal equilibrium with temperature $T$ and prepared in the same double-well trap (see Supplemental Material [24]), we observe $\lambda_{\phi} \propto$ $1 / T$ (green circles in Fig. 4), emphasizing the different characteristic scalings of the thermal and prethermalized phase-correlation lengths. We finally note that the crossover length scale $l_{0}$ determines the prethermalization time scale $\left(\sim 10 \mathrm{~ms}\right.$ in our experiments) through its ratio $l_{0} / c$ with the speed of sound in the system [21].

In conclusion, we demonstrated the multimode nature of the dynamics of a coherently split 1D Bose gas using matter-wave interferometry. Our approach allows the direct observation of the effect of the quantum noise present in the initial nonequilibrium state on the evolution of the manybody system. We showed the emergence of two distinct regimes of relaxation separated by a characteristic crossover length scale, which we identified as the prethermalized phase-correlation length of the system. This characteristic length scale $2 \hbar^{2} / \mathrm{mg}$ reflects the parametrization of the many-body theory describing the dynamics of the system by a single parameter $g$. For our closed system to thermalize and the relative-phase correlation length to reach the true thermal equilibrium value $\lambda_{\phi}$, the integrability of the LL theory needs to be broken. Investigations into the processes leading to the full thermalization of the system are ongoing.

We acknowledge fruitful discussions with Igor Mazets. Our work was supported by the Austrian Science Fund (FWF) through the Wittgenstein Prize and the EU through the integrating project AQUTE. M.K., T.L., and M.G. thank the FWF Doctoral Programme CoQuS (W1210), R.G. is supported by the FWF (Lise Meitner project M1423), and D.A.S. acknowledges the EU (Grant No. 220586). T. K. and E. D. thank DARPA, HarvardMIT CUA, NSF (Grant No. DMR-07-05472), and AROMURI on Atomtronics.

*schmiedmayer@atomchip.org

[1] A. Arrizabalaga, J. Smit, and A. Tranberg, Phys. Rev. D 72, 025014 (2005).

[2] P. Arnold, J. Lenaghan, G. D. Moore, and L. G. Yaffe, Phys. Rev. Lett. 94, 072302 (2005).

[3] A. Rebhan, P. Romatschke, and M. Strickland, Phys. Rev. Lett. 94, 102303 (2005).

[4] D. Podolsky, G. N. Felder, L. Kofman, and M. Peloso, Phys. Rev. D 73, 023501 (2006).

[5] T. Micklitz and A. Levchenko, Phys. Rev. Lett. 106, 196402 (2011).

[6] J. Marino and A. Silva, Phys. Rev. B 86, 060408 (2012).

[7] M. A. Cazalilla and M. Rigol, New J. Phys. 12, 055006 (2010).

[8] A. Polkovnikov, K. Sengupta, A. Silva, and M. Vengalattore, Rev. Mod. Phys. 83, 863 (2011).

[9] T. Kinoshita, T. Wenger, and D. S. Weiss, Nature (London) 440, 900 (2006).

[10] M. Rigol, V. Dunjko, V. Yurovsky, and M. Olshanii, Phys. Rev. Lett. 98, 050405 (2007).

[11] M. Kollar, F. A. Wolf, and M. Eckstein, Phys. Rev. B 84, 054304 (2011).

[12] M. Gring, M. Kuhnert, T. Langen, T. Kitagawa, B. Rauer, M. Schreitl, I. Mazets, D. Adu Smith, E. Demler, and J. Schmiedmayer, Science 337, 1318 (2012).

[13] S. Hofferberth, I. Lesanovsky, T. Schumm, A. Imambekov, V. Gritsev, E. Demler, and J. Schmiedmayer, Nat. Phys. 4, 489 (2008).

[14] T. Kitagawa, A. Imambekov, J. Schmiedmayer, and E. Demler, New J. Phys. 13, 073018 (2011). 
[15] J. Berges, S. Borsányi, and C. Wetterich, Phys. Rev. Lett. 93, 142002 (2004).

[16] C. Kollath, A. M. Läuchli, and E. Altman, Phys. Rev. Lett. 98, 180601 (2007).

[17] M. Moeckel and S. Kehrein, Phys. Rev. Lett. 100, 175702 (2008).

[18] M. Eckstein, M. Kollar, and P. Werner, Phys. Rev. Lett. 103, 056403 (2009).

[19] L. Mathey and A. Polkovnikov, Phys. Rev. A 81, 033605 (2010).

[20] R. Barnett, A. Polkovnikov, and M. Vengalattore, Phys. Rev. A 84, 023606 (2011).

[21] R. Bistritzer and E. Altman, Proc. Natl. Acad. Sci. U.S.A. 104, 9955 (2007).

[22] Atom Chips, edited by J. Reichel and V. Vuletic (Wiley VCH, Berlin, 2011).

[23] T. Schumm, S. Hofferberth, L. M. Andersson, S. Wildermuth, S. Groth, I. Bar-Joseph, J. Schmiedmayer, and P. Kruger, Nat. Phys. 1, 57 (2005).

[24] See Supplemental Material at http://link.aps.org/ supplemental/10.1103/PhysRevLett.110.090405 for a description of the quasi-1D Bose gas parameters, the splitting process, the extraction of the contrast and comparison to theory, and of the estimation of the relative phase correlation length.

[25] D. A. Smith, S. Aigner, S. Hofferberth, M. Gring, M. Andersson, S. Wildermuth, P. Krüger, S. Schneider, T. Schumm, and J. Schmiedmayer, Opt. Express 19, 8471 (2011).

[26] T. Giamarchi, Quantum Physics in One Dimension, International Series of Monographs on Physics (Clarendon Press, Oxford, 2004).

[27] T. Kitagawa, S. Pielawa, A. Imambekov, J. Schmiedmayer, V. Gritsev, and E. Demler, Phys. Rev. Lett. 104, 255302 (2010).
[28] A. Widera, S. Trotzky, P. Cheinet, S. Fölling, F. Gerbier, I. Bloch, V. Gritsev, M. Lukin, and E. Demler, Phys. Rev. Lett. 100, 140401 (2008).

[29] A. Polkovnikov, E. Altman, and E. Demler, Proc. Natl. Acad. Sci. U.S.A. 103, 6125 (2006).

[30] N. K. Whitlock and I. Bouchoule, Phys. Rev. A 68, 053609 (2003).

[31] T. Betz, S. Manz, R. Bücker, T. Berrada, C. Koller, G. Kazakov, I. E. Mazets, H.-P. Stimming, A. Perrin, T. Schumm et al., Phys. Rev. Lett. 106, 020407 (2011).

[32] S. Manz, R. Bücker, T. Betz, Ch. Koller, S. Hofferberth, I. E. Mazets, A. Imambekov, E. Demler, A. Perrin, J. Schmiedmayer et al., Phys. Rev. A 81, 031610(R) (2010).

[33] The choice of integration lengths to small scales is limited by the resolution of our imaging system $(3.8 \mu \mathrm{m}$, see Supplemental Material [24]) and to large scales by the longitudinal size of the cloud $(\sim 100 \mu \mathrm{m})$. We choose $6 \mu \mathrm{m}$ for the smallest length scale to be clearly above the resolution limit. The intermediate length scales are selected arbitrarily to illustrate the smooth crossover between the two regimes.

[34] Our model assumes an infinitely fast splitting and predicts a contrast of $100 \%$ at $t=0$. The finite time splitting used in the experiment results in an uncertainty of about $0.5 \mathrm{~ms}$ on the point in time where the two quasicondensates decouple, where fast dephasing of high-energy excitations contributes to a reduction of the contrast. Moreover, technical imperfections reduce the contrast, mainly the residual projection of atoms to other Zeeman states during the switch off of the trapping potential. On all length scales, the maximum contrast that we detect (taking into account finite resolution) is approximatively $85 \pm$ $3 \%$, in line with the factor of $r=0.74$ used in Fig. 3 to normalize the theory data for the mean squared contrast $\left(0.85^{2} \approx 0.72\right)$. 